\title{
Design of E and U Shaped Slot for ISM Band Application
}

\author{
M. Paranthaman and S. Palanivel Rajan
}

Department of Electronics and Communication Engineering, M. Kumarasamy College of Engineering, Karur, Thalavapalayam, Karur - 639113, Tamil Nadu, India; paranthaman765@gmail.com, drspalanivelrajan@gmail.com

\begin{abstract}
Objectives: For implantable devices, a miniaturized antenna structure is proposed to operate in ISM band. At 2.5 $\mathrm{GHz}$ frequency the antenna is expected to operate. Methods/Statistical Analysis: A combination of E and U shaped slots on a rectangular patch design is proposed for biomedical applications especially operating in ISM band. The slots created here in order to changing the current path so that the radiation pattern acquired in excepted frequency. Findings: The E and U slots on a rectangular patch operates at $2.5 \mathrm{GHz}$ and it provides good radiation pattern and gain. Applications/Improvements: Antennas dipped inside a human body are highly required for biotelemetry applications. Careful design of antenna is needed since the antennas are operated inside human body. The proposed structure gives good commitment towards the requirements.
\end{abstract}

Keywords: Biotelemetry, E Shape, Implantable Antenna, ISM Band, U Shape

\section{Introduction}

In each and every activities of human life the advancement of wireless communications plays a vital role. Body centered communication devices are the major research area in future. Unlike off-body or on body, inbody devices have high level of complexity and poor environment. These in-body devices have the potential to wirelessly communicate to devices which are monitored continuously. The requirements of implantable radiating structure are different from free space antennas. Since the device is going to place inside human body the first and foremost requirement is the antenna size and also it should be compatible with human tissues. Since it is transmitting wirelessly to external device power consuming capability and life time of the device can be added to requirements. Miniaturization of the implantable device can be accomplished by using CMOS technology. This is because of the recent developments in integrated circuits. Dielectric substrate with high permittivity, increase in radiating path and perfect matching of impedance are the some of the methods to lessen the volume of the radiating device $\mathrm{e}^{\mathrm{1-5}}$.

Patient safety is the major consideration because human tissues may be in conductive nature, and it can short circuit the radiating structure if it is permitted to direct contact. There are two ways followed by researchers to avoid unwanted short circuits are made up the antenna on biocompatible material and coat the radiating element with biocompatible elements. Such materials are ceramic alumina and Teflon. If implantable devices are used continuously the transceiver of the device will lose some energy and it leads to reduction of the lifetime of the device ${ }^{6}$. To avoid this, the device may be configured with dual band operation so that it can be operated in one band and put on sleep in another band. Inside the skin or muscle, the antenna is highly responsive to the environment and the properties of immersed antennas are severely affected, careful selection of material will reduce the undesired effects created by the human body. 
Instead of immerse the antenna by operating; ingestible antennas are the advanced addition in the field of body centered wireless communication.

For excellent communication link establishment between immersed antennas to the external device, the antenna must produce good radiation efficiency. There were two skin models used in recent researchers are one layer and three layer. Since multi layer of tissues in human body has different dielectric properties, the calculations related to layers must be handled carefully. Major design issues related to tissue model are absorbing nature of RF signals, reflections and interfaces, matching of antenna with tissues ${ }^{?}$.

$\mathrm{In}^{1}$, a novel double band implantable reception apparatus with differentially bolstering is proposed. The planned receiving wire has created on substrate and covered by superstrate and both are made by Rogers 6010 . Superstrate utilized here is to keep the receiving wire from undesired contact with directing tissues of human body. Double encouraging to the radio wire is presented in this examination, with the goal that the transmitting structure must be symmetrical. Winding shape two bolstering radio wire demonstrates some change consequently misfortune. The thickness of the tissue can be fluctuated and again the receiving wire can be tried to know the double band task the proposed plan.

$\mathrm{In}^{2}$, for implantable gadgets a double band receiving wire is proposed. This examination utilizes substrate and superstrate made by Rogers 3010. By shorting pin of the winding resonator double band task is accomplished.

$\mathrm{In}^{3}$, double band reception apparatus with differential bolstering is proposed with in vitro testing. This exploration portrays about the reenacting condition with regards to one layer and three-layer tissue show. Cubic state of the apparition is utilized here for testing. Substrate utilized is Rogers 3010 and there is no utilization $\mathrm{f}$ superstrate here not at all like past explores recorded previously. Rather than superstrate, the other method for protecting strategy is utilized here. Parylene- $\mathrm{C}$ is a biocompatible material covered the planned receiving wire.

$\mathrm{In}^{4}$, double band implantable receiving wire is proposed. Rogers's 3010 substrate utilized here. Parylene-C is a biocompatible material covered the composed radio wire. The protecting material lessens the power dispersal because of undesirable short out impact made. Dipole radio wire alongside winding patch gives double band activity here.

\section{Antenna Design}

The goal this proposed work is to design a small size implantable antenna operating in ISM band. Figure 1 shows the three dimensional perception of the proposed structure with a substrate Rogers $3010\left(\varepsilon_{\mathrm{r}}=10.2\right)$ and the height of the substrate is $0.635 \mathrm{~mm}$. When we correlate the previous researches $1265.6 \mathrm{~mm}^{35}, 1322.5 \mathrm{~mm}^{32}$-and $691.515 \mathrm{~mm}^{34}$ with the proposed antenna, the size is minimum $19 \mathrm{~mm} \times 26.4 \mathrm{~mm} \times 0.635 \mathrm{~mm}\left(318.5 \mathrm{~mm}^{3}\right)$.

On a rectangular fix, first the $\mathrm{E}$ formed space has been made with the length and width of $1 \mathrm{~mm}^{1,2}$. To produce capacitive effect on the patch the $\mathrm{U}$ slot has been created so as the radiating element may be produce good radiation on the desire direction ${ }^{3}$.

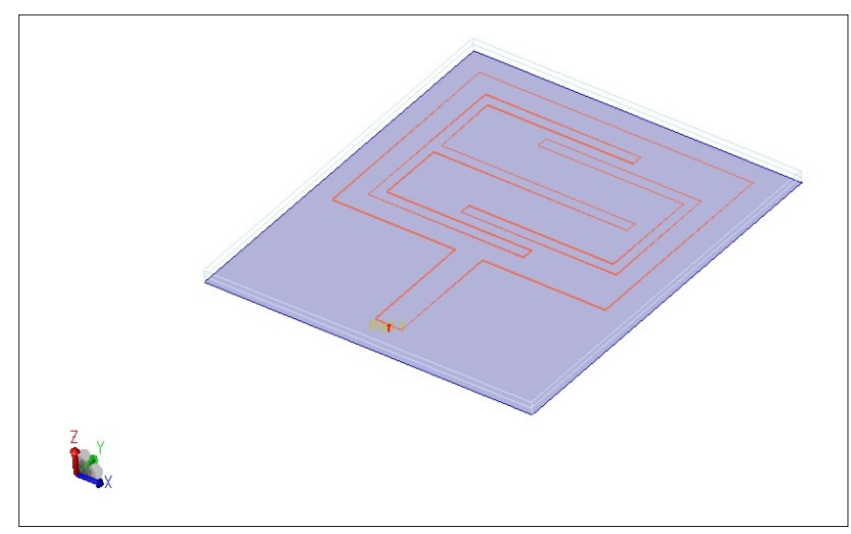

Figure 1. Proposed antenna.

\section{Result Analysis and Discussions}

Return loss of any patch antenna must be less than $-10 \mathrm{~dB}$. The proposed antenna possesses $-24.37 \mathrm{~dB}$ at radiating frequency which is displayed in Figure 2. The Simulated gain of $-35 \mathrm{~dB}$ acquired at working ISM band recurrence displayed in Figure 3. The pattern of radiating element of proposed patch is shown in Figure 4 and it shows the power radiated bidirectional at 45 degrees. When compared to $\frac{1,3,5}{5}$ the proposed antenna gives good commitment to achieve the desired operation. Since only the simulated results are displayed here, the measurement of this structure with an insulating layer (using biocompatible material) can be done as future work. 


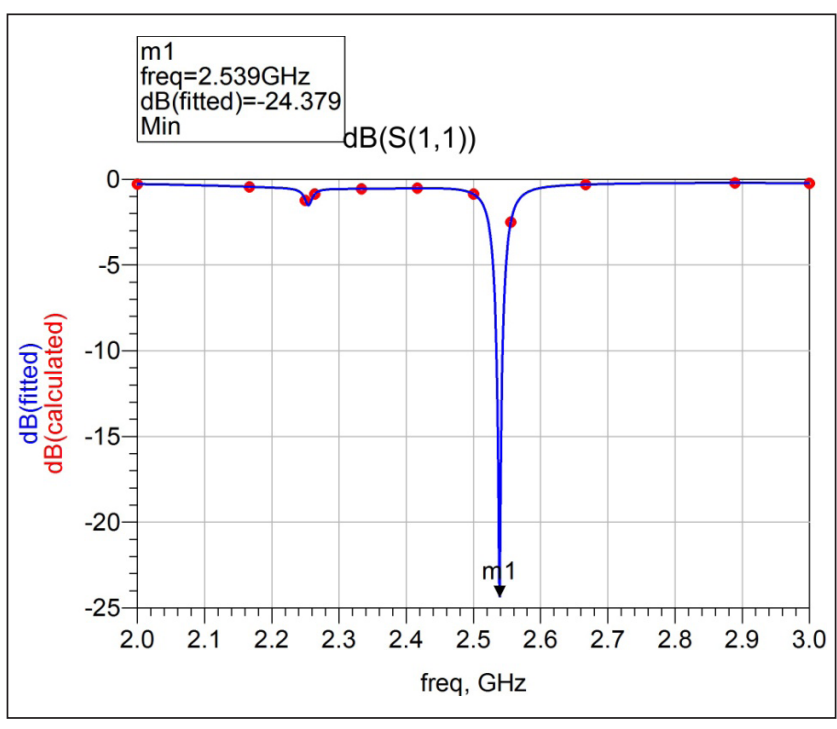

Figure 2. Return Loss $\left(S_{1,1}\right)$ in $\mathrm{dB}$.

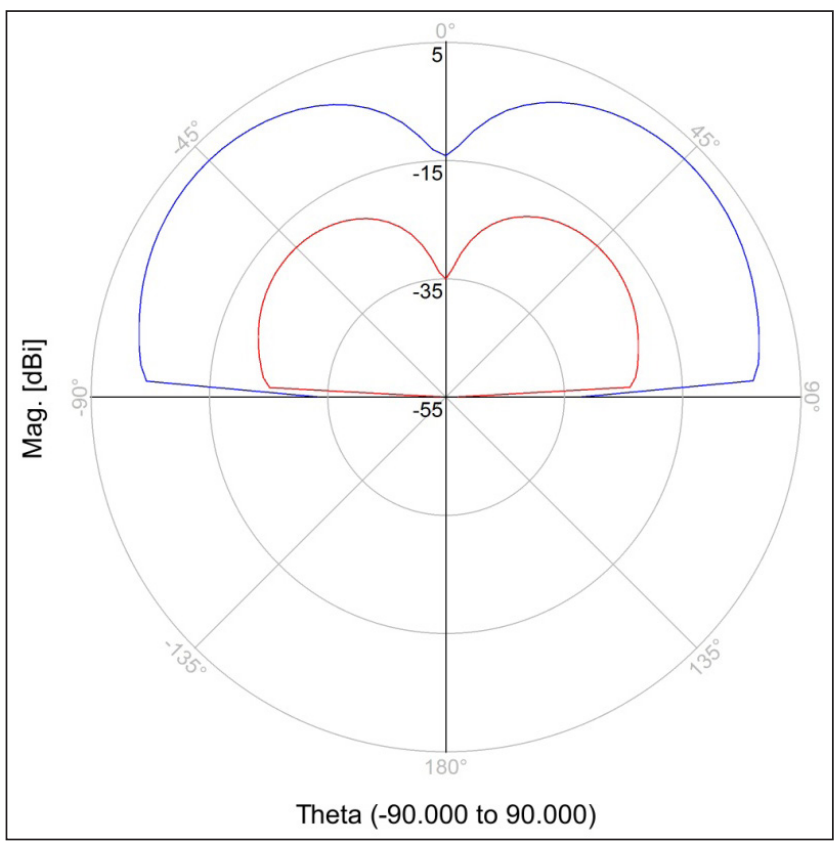

Figure 3. Gain and directivity.

\section{Conclusion}

A miniaturized rectangular patch with a combination of $E$ and $U$ shaped slot is demonstrated for ISM band application. The Correlation between the proposed designs with previous researches is given. When compared to previous works, this antenna is small in size $\left(318.5 \mathrm{~mm}^{3}\right)$. At the resonant frequency the proposed design shows good

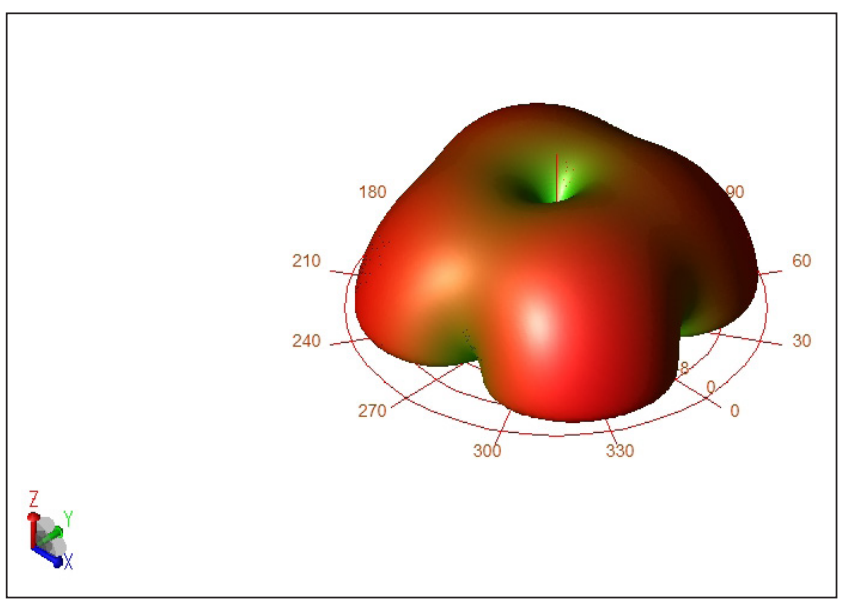

Figure 4. Radiation pattern.

radiation pattern and return loss of $-24 \mathrm{~dB}$. However, the proposed system can be modified to operate in MICS band also in future.

\section{References}

1. Duan Z. Differentially fed dual-band implantable antenna for biomedical applications, IEEE Transactions on Antennas and Propagation. 2012; 60(12):5587-95. Crossref.

2. Paranthaman M, Vijayprasath S, Rajan SP. Design of a frequency tunable patch antenna using HFSS, International Journal of Advanced Research Trends in Engineering and Technology. 2016; 3(7):69-72.

3. Liu C. Compact dual-band antenna for implantable devices, IEEE Antennas and Wireless Propagation Letters. 2012; 11:1508-11. Crossref.

4. Karacolak T, Hood AZ, Topsakal E. Design of a dualband implantable antenna and development of skin mimicking gels for continuous glucose monitoring, IEEE Transactions on Microwave Theory and Techniques. 2008; 56(4):1001-08. Crossref.

5. Karacolak T, Cooper R, Topsakal E. Electrical properties of rat skin and design of implantable antennas for medical wireless telemetry, IEEE Transactions on Antennas and Propagation. 2009; 57(9):2806-12. Crossref.

6. Paranthaman M, Shanmugavadivel G. Design of frequency reconfigurable e-shaped patch antenna for cognitive radio, International Journal of Applied Engineering Research. 2015; 10(20):16546-48.

7. Paranthaman M. T-shape polarization reconfigurable patch antenna for cognitive radio. In: Third International Conference on Science Technology Engineering and Management (ICONSTEM); 2017. p. 927-92. Crossref. 